\title{
Article
}

\section{Prospective, longitudinal assessment of quality of life inpatients with cancer of the head and neck and their primary carers}

Terro, W and Crean, Stjohn

Available at http://clok.uclan.ac.uk/17821/

Terro, W and Crean, Stjohn ORCID: 0000-0001-9336-8549 (2017) Prospective, longitudinal assessment of quality of life inpatients with cancer of the head and neck and their primary carers. British Journal of Oral and Maxillofacial Surgery . ISSN 0266-4356

It is advisable to refer to the publisher's version if you intend to cite from the work. https://doi.org/10.1016/j.bjoms.2017.04.004

For more information about UCLan's research in this area go to http://www.uclan.ac.uk/researchgroups/ and search for <name of research Group>.

For information about Research generally at UCLan please go to http://www.uclan.ac.uk/research/

All outputs in CLoK are protected by Intellectual Property Rights law, including Copyright law. Copyright, IPR and Moral Rights for the works on this site are retained by the individual authors and/or other copyright owners. Terms and conditions for use of this material are defined in the policies page. 
Title:

PROSPECTIVE, LONGITUDINAL BASELINE ASSESSMENT QUALITY OF

LIFE STUDY IN HEAD AND NECK CANCER PATIENTS

AND THEIR PRIMARY CARERS Key words: Head and neck cancer; quality of life; primary carer; assessment;
Impact

\begin{abstract}
:
Head and neck cancer H\&N Ca has profound psychosocial and physical impacts upon patients. Thus, quality of life QOL is an essential consideration - not only is it of importance to the patient but also provides a subjective measure of success. Little work has been done on the influence of this disease on the carers. The aim of this longitudinal study was to assess the impact of H\&N Ca diagnosis and treatment on the QOL of patients and their carers from baseline to three months. Methods; 36 patients with a diagnosis of H\&N Ca and 21 primary carers were enrolled. Patients completed one H\&N specific measure, the University of Washington H\&N Version Four; UWV4 and three global questionnaires at the diagnosis, one and three months postoperatively. The carers completed similar questionnaires except for UW- V4. Results; Analysis of patient's data showed a statistically significant deterioration in psychosocial and physical domains at one month postoperatively $(\mathrm{p}<0.05)$. However, the analysis of carers data showed a statistically significant deterioration $(\mathrm{p}<0.01)$ in anxiety and depression domains which remained low at 3 months follow-up. Conclusion; These findings highlight the need for psychological support of not only patients but also their primary carers during management of H\&N Ca.
\end{abstract}




\section{Introduction}

In head and neck cancer, quality of survival is critically influenced by performance or functional ability in areas such as eating, swallowing, and speaking ${ }^{1,2}$. Eighty percent of oral cancers are squamous cell carcinoma $\mathrm{SCC}^{2,3}$. The three main modalities of head and neck cancer treatment are surgery, radiotherapy, and / or chemotherapy used alone or in combination ${ }^{4,5,6 .}$ The quality of life following treatment for head and neck cancer is an area of increasing interest to the individual patient. Quality of life in head and neck cancer clinical trial require measures that are relatively comprehensive, short, concise, and easy to understand ${ }^{8,9 \text {, and } 11}$. Four main categories are currently in usage, with special reference to head and neck cancer, they are global, global cancer, head and neck specific, and performance ${ }^{7,10}$. Hence, it is an important tool for evaluating outcome in conjunction with mortality, morbidity, and survival and recurrence rate. General satisfaction with life has been demonstrated in many studies, while others revealed the opposite effects ${ }^{12,13,14}$

The changes that the patients experience as a result of the head and neck cancer and subsequent treatment are often associated with negative influences not only on the patient quality of life but also on the primary carers (father, mother, wife,...) ${ }^{19,20,21}$.

Although, the general QOL has been assessed in some studies of head and neck cancer patients, little work has been done to assess the impact that disease treatment has upon the patient and the patient's primary carers using different types of questionnaires ${ }^{2,3,19,20 \text {,and } 21 .}$ 
The aim of this study is to assess in detail the impact that the current treatment option has upon patients and their carer's quality of life from baseline to three months.

\section{Materials and Methods:}

Study participants were consecutive new patients and their carers, underwent surgery for previously untreated head and neck cancer attending the Head and Neck clinic (starting September 2002 to December 2002) where patients from the Department of Oral and Maxillofacial Surgery, and Ear, Nose and Throat at the University Hospital of Wales had been approached. Demographic variables of interest included age, sex, occupation, and personal contact number, histology type of tumour, relevant past medical history, and present treatment plan. All the patients with a confirmed diagnosis of head and neck cancer underwent complex treatment included subsequent resection and reconstruction under general anaesthesia. Patients with lymphoma or skin cancer in the head and neck region along with patients receiving radiotherapy or/and chemotherapy were excluded from this study. When informed consent had been obtained the patients were given four questionnaires: the University of Washington Head and Neck Disease- Specific Questionnaire Quality of Life Version Four UW-QOL V4, the Medical Outcomes Study- Short Form 36 SF-36, the Hospital Anxiety and Depression Scale HADS, the Davidson Trauma Scale DTS 15, 16, 17, and 18 . Patients were asked to complete the questionnaires before surgery, at one month, and three month after surgery. The majority of patients were approached directly at the clinics. Those patients, who failed to attend, were sent the questionnaires and asked to complete them and return in a stamped addressed envelope. No further questionnaires were given to patients who developed recurrent disease during the follow- up period. 
To assess the impact the diagnosis and treatment had upon the primary carers, the individual identified was asked to complete three of questionnaires: HADS, SF-36, and DTS.

Ethical approval was obtained for this study by the Bro Taf Health Authority, Local Research Ethics Committee, Temple of Peace and Health.

Statistical analysis was descriptive, through the use of tables and summary statistics. For descriptive purposes we used Mean (m), and Standard Deviation SD. Data was analysed using parametric (paired samples statistics, T-Test) and non-parametric Wilcoxon Signed Ranks Test methods. We highlighted correlation significant at less than $0.05(\mathrm{p}<0.05)$ and highly significant at less than $0.01(\mathrm{p}<0.01)$ levels in the results. 


\section{Results:}

\section{Patient's sample;}

A total of 36 patients were included in the study. The mean age was 78.5 years (range 41years to 88years). There were 24 male and 12 female patients with a male to female ratio 2:1. 25 (69.4\%) were retired, 7 (19.40\%) unemployed, 2 (5.50\%) employed and 2 (5.50\%) homemakers. The majority of patients were smokers 33 (91.6\%), whereas $33(91.6 \%)$ were alcohol consumers. The most common sites of tumour involvement were the larynx $24.9 \%(9 / 36)$, buccal mucosa $2.7 \%$ (1/36), floor of the mouth $11 \%$ (4/36), tonsils 5.5\% (2/36), parotid glands $11 \%$ (4/36), oral tongue $13.8 \%(5 / 36)$, alveolar mucosa $8.2 \%(1 / 36)$, and neck 5.5\% (2/36).

Five patients discontinued the study: one could no longer fill the questionnaires at the postoperative period (1/12 \& 3/12), one discontinued for unknown reasons and three patients received radiotherapy and/or chemotherapy alone, having completed preoperative questionnaires. The number of patients who completed all four questionnaires at preoperative period was 36. In comparison, those completed at the one month and three month postoperative period were 33 and 31, respectively. The results from UW-QOL V4, SF -36, and HADS are revealed in Table 1, 2, and 3.

The Davidson Traumatic Scale was used as a self-rating scale to measuring symptom severity in post-traumatic stress disorder PTSD. Preoperatively, the number of patients on frequency scale $(0=$ Not at all) was 13 , which reduced to 4 at three months follow-up, whereas the number of patients on the scale $>/=2$ decreased from 23 to 20 patients. Similar changes were noted on the severity scale measures. When the results from the three months follow-up were compared with the pre-operative results $(0 / 12)$, 
the number of patients with extremely distressing symptoms decreased from 20 to 15 and those recording not at all distressing reduced from 11 to 8 . The number of patients with moderately distressing was constant throughout the study. The most notable changes were on the severity scale measure. When the results from the three months follow-up were compared with the results at the preoperation stage, the number of patients with extremely distressing symptoms decreased from 20 to 15 patients, and not at all distressing from 11 to 8 . The number of patients with moderately distressing was constant throughout the study. 


\section{Primary carer's sample;}

21 primary carer were recruited, 5 (23\%) men and 16 (76.1\%) women. One person was dropped out for unknown reasons? There was a female to male ratio of 3.2:1. The mean age was 52.4 years, (Range 32 to 74years); in regards to occupation, 23\% (5/21) were employed, 52.3\% (11/21) retired and 23.8\% (5/21) were homemaker.

All the participants had answered three different type of questionnaires, SF-36, HADS and DTS. The most significant changes in the SF-36 were noted for physical problems (physical function and role function) and social functioning. The mean score for physical functioning decreased from 22.3 to 20.3 (best value $=30, \mathrm{p}<0.01$ ); the mean score for role limitation physical, decreased from 6.9 to 4.8 (best value $=8$. $\mathrm{p}<0.01$ ). The results from SF-36 are shown in Table 4. The HADS was used to assess psychiatric disorders among the primary carers. The number of patients scoring as probable cases of anxiety and depression, pre-operatively, was 9 (42.8\%) and 2 (10\%) respectively. By three months the number of probable anxiety cases had reduced to 4 (20\%), whereas the number of probable depression cases had increased to 6(30\%). The results from the HADS are shown in Table 5. On the frequency scale of DTS, the number of patients scoring 0 (not at all) decreased from 6 (28.5\%) at 0/12 to 2 (10\%) at 3 months. The number of patients scoring $>/=2$ increased from 15 (71.4) to 18 (90\%). On the severity scale there was a decrease from $12(57 \%)$ to $6(30 \%)$, in the number of cases scoring extremely distressing status, by the three months follow-up stage. In contrast the number of markedly distressing cases increased from 0 to 5 (25\%). An increase in the moderately distressing cases from 2 (9.5\%) to 5 (25\%) was also noted over the same period. The results from the DTS are demonstrated in Table 6. 


\section{Discussion;}

The measurement of performance status of head and neck cancer patients is an important consideration in the evaluation of treatment outcomes and rehabilitation strategies. Traditionally, disease control and survival have been the measures of treatment efficacy. Today however with increasing disease free survival rates, the psychosocial dimensions of cancer care and the quality of survival have become areas of concern. This study was a prospective, short-term study in which quality of life was evaluated before and after of surgical treatment in patients with head and neck cancer. It also assessed the impact that the disease and disease related treatment had upon the patient’s primary carer.

A total of 36 patients and 21 primary carers were included in the study. Five patients discontinued, two died and three disrupted the study. One carer dropped out. The male to female ratio, was 2:1. It was noted that the majority of the patients were smokers and consumed alcohol. Similar results in regards male to female ratios, smoking, and alcohol consuming have been found in other study A. Chandu. 2002. The mean age of the study group was 78.5 years. This is compared favourably with the studies of S.A. Abdelkarim.2000, E. Hammerlid, et al. 1997. The group are relatively elderly but did not influence the results of most of the questionnaires.

In this study, most of the tumours were squamous cell carcinoma. The common tumour sites were the larynx $24.9 \%(9 / 36)$ and tongue $13.8 \%(5 / 36)$.

A quality of life outcomes monitoring system for head and neck cancer requires tools that is not only valid and reliable but that address broad constructs of general health as well as those specific to the head and neck region. Therefore, the combination of specific and general measures provides the most comprehensive assessment strategy. 
Four questionnaires were given to the patients: UW-QOL V4, SF-36, HADS, and DTS. Three questionnaires SF-36, HADS, and DTS were given to the primary carer's group. In 1996, S.J. Hassan, G.I. Zimmerman, looked at seventy-five patients with surgically treated head and neck cancer. The patients, completed QOL questionnaire UW-QOL V4 before surgery, immediately following surgery, and three months postoperatively. The survey UW-QOL V4 assessed pain, disfigurement, activity, recreation, chewing, swallowing, speech, and shoulder disability. They found that the majority of patients reported that their overall QOL was less than excellent. Pain interfering with daily activities was reported by approximately one third of subjects, with reported impact on social activities. Our study was similar but with the addition of the SF-36 questionnaire. We have shown that the commonest complaints in the UW-QOL V4 questionnaire pre-operatively were pain, anxiety, activity and mood whereas the most notable complaints in the SF-36 questionnaire ware also pain and vitality. In comparison with Hassan et al. who showed pain was reported by approximately one third of the patients to interfere with daily activities, our study showed similar findings in two thirds. Pain was the main feature in both studies.

When we compared the results between one month, and three months follow-up, we had found quite a bit improvement in both UW-QOL V4 and SF-36 scores. The two pain scales in UW-QOL V4 and SF-36, suggest the same outcome, namely of very slow improvement to three months. A longitudinal study done by Rogers SN 1998 showed that pain on the three scales UW-QOL V4, SF-36 showed a slow improvement to three and six months, and then was quite a bit improvement by twelve months. This result suggested our hypothesis about improvement on pain scale 
over time. Not unexpectedly, pain, functional ability (swallowing, chewing, speech, etc) and social functioning problems were the most common related treatment symptoms that interfered with patient's daily life after surgery.

We include the HADS (S.N. Rogers 1999) and DTS (J.R. Davidson 2002) in this study because they had been shown to screen for psychiatric disorders. They revealed a high degree of psychological problems indicating psychiatric disorders in a great number of patients at the preoperative period. Considerable variability in the level of recovery was observed at three months follow-up. This finding is in agreement with a previous prospective, longitudinal quality of life study of patients with head and neck cancer (E. Hammerlid 1997). Further investigation is needed to determine if the improvement for psychological distress and anxiety has stopped developing over time. A study done by K. Bjordal 1995, confirmed that psychiatric distress persisted long after successful treatment.

When the results of DTS at preoperation were compared with the results at one month, and three months follow-up, there was a tendency toward decreasing measures of severity and frequency scales. The corresponding results from the two different questionnaires (HADS, and DTS), revealed that most deterioration of psychological aspects was found before and after surgery, whereas a remarkable improvement can be regarded at three months follow-up.

Little is known however, about the impact that the disease has upon the primary carer. A total number of twenty-one carers (5 men, 16 women) with an average of 52.6 years and female to male ratio 3.2:1 were included in the study. One carer left the study with unknown reasons. Changes that the patients experienced on their life as a result of the illness and subsequent treatment, also affected the life of their carers. When we compared the results between preoperative period and three months follow- 
up, we found that most of the domains (physical functioning, role limitation functioning and social functioning) of the SF-36 questionnaire showed a deterioration in the scores obtained. In this study the primary carer was found to report high degree of psychological disorders especially at post-operative period. It therefore seems likely that the carer's group, supported the patients throughout their illness were more anxious and depressed at pre-operative and post-operative time than the patients themselves. We might state that the reaction of the primary carer have had an impact on some of the results obtained from the patients regarding the scores obtained from most of the questionnaires.

Limitations of this study include that it was small short term study with relatively old patients. This limits the conclusions that can be drawn from the findings to some of head and neck cancer domains. 


\section{References}

1. Beeken L, Calman F.

A return to normal eating after curative treatment for oral cancer

What are the long-term prospects?

Euro J Cancer B Oral Oncol 1994; 30: 387- 92.

2. Aaronson NK.

Assessing quality of life (QL) in cancer patient trials

European J of Cancer 2001; 37: 57-58.

3. Sammy J, Hassan MD, Weymuller EA.

Assessment of quality of life in Head and Neck Cancer patients

Head and Neck 1993, 15: 485-496.

4. Daniel T, Pavel D, Abdelkarim S. A.

Salvage surgery after radical accelerated radiotherapy with concomitant boost technique for head and neck carcinomas

Head \& Neck 2005, 27(3): 182-186.

5. Allal AS, Dulguerov P, Bieri S, Lehmann W, Kurtez JM.

Assessment of quality of life in-patients treated with accelerated radiotherapy for laryngeal and hypopharyngeal carcinomas

Head \& Neck 2000, 3:288-93.

6. Allen CS, Stephanie S, Dawn CA, Emre V, Brian O, Ehab H. Assessing quality of life in patients with head and neck cancer 
Arch Otolaryngol Head Neck Surg 2000; 126:459-467.

7. Bjordal K, Kassa S.

Psychological distress in head and neck cancer patients 7-11 years after curative treatment

Br J Cancer 1995; 71: 592-7.

8. Bjordal K, Ahlener-Elmqvist M, Tollesson E.

Development of a European Organisation for Research and Treatment of

Cancer questionnaire module to be used in quality of life assessment in

head and neck cancer patients

Acta Oncol 1994; 28:847-56.

9. Rogers SN, Fisher SE, Woolgar JA.

A review of quality of life assessment in oral cancer

Int J Oral Maxillofac Surg 1999; 82: 99-117

10. Rogers SN, Lowe D, Brown JS, Vaughan ED.

A comparison between the UW-QOL and SF-36, EORTC QOQ-C33 and EORTC Head and Neck 35 J Oral Oncology 1998, 34: 361-372

11. Davies ADM, Davies C, Delpo MC.

Depression and anxiety in patients undergoing diagnosting investigations for head and neck cancers Br J Psychiatry 1986, 149: 491-3. 
12. Bjordal K, Kassa S.

Patient self-reported and clinician-rated quality of life in head and neck cancer patients: a crosssectional study

Euro J Cancer B Oral Oncol 1995; 31: 235-41.

13. Chatura SK, Shenoy SM, Premlatha KM.

Concerns, coping and quality of life in head and neck surgery patients

Support Care Cancer 1996; 4: 186-90.

14. Hassan SJ, Weymuller EA.

Assessment of quality of life in head and neck cancer patients

Head Neck 1993; 15: 485-496.

15. Hammerlid E, Person OL, Westin T.

Quality-of-life effects of psychosocial intervention in patients with Head and Neck Cancer Otolaryngol Head Neck Surg 1999; 120: 507-516.

16. Zigmond AS, Snaith RP.

The hospital Anxiety and Depression Scale

Acta Psychiatr Scand 1983; 67: 361-370.

17. E Hammerlid, E Silander, RN Hoornestam, M Sullivan

HEALTH-RELATED Quality of life three years after diagnosis of head and neck

cancer- A longitudinal study

Head \& Neck 2000; 23: 113-125. 
18. Davidson JR, Tharwani HM.

Davidson Trauma Scale (DTS): normative scores in the general population and effect sizes in Placebo-controllled trials

Depress Anxiety 2002; 15: 75-8

19. Lais R, Raphaell FB, Rafael DC, Jossi LK, Leandro LM.

Quality of life impairment in patients with head and neck cancer and their caregivers: a comparative study

Brazilian j Otolaryngol 2016; 82: 680-686.

20. Lin CR, Chen SC, Chang JT, Fang YY, Lai YH.

Fear of cancer recurrence and its impact on quality of life in family caregivers of patients with head and neck cancers

J Nurs Res 2016; 24(3): 240-8.

21. Hodges LJ, Humphris GM.

Fear of recurrence and psychological distress in head and neck cancer patients and their carers

Psychooncology. 2009; 18(8):841-8. 